\title{
Planning and scheduling of semi-urgent surgeries
}

\author{
Maartje E. Zonderland • \\ Richard J. Boucherie • Nelly Litvak • \\ Carmen L. A. M. Vleggeert-Lankamp
}

Received: 7 July 2009 / Accepted: 19 February 2010 / Published online: 19 March 2010

(C) The Author(s) 2010. This article is published with open access at Springerlink.com

\begin{abstract}
This paper investigates the trade-off between cancellations of elective surgeries due to semi-urgent surgeries, and unused operating room (OR) time due to excessive reservation of OR time for semi-urgent surgeries.Semi-urgent surgeries, to be performed soon but not necessarily today, pose an uncertain demand on available hospital resources, and interfere with the planning of elective patients. For a highly utilized OR, reservation of OR time for semi-urgent surgeries avoids excessive cancellations of elective surgeries, but may also result in unused OR time, since arrivals of semiurgent patients are unpredictable. First, using a queuing theory framework, we evaluate the OR capacity needed to accommodate every incoming semi-urgent surgery. Second, we introduce another queuing model that enables a trade-off between the cancelation rate of elective surgeries and unused OR time. Third, based on Markov decision theory, we develop a decision support tool that assists the scheduling process of elective and semi-urgent surgeries. We demonstrate our results with actual data obtained from a department of neurosurgery.
\end{abstract}

M. E. Zonderland ( $\varangle)$

Division I, Leiden University Medical Center, Postbox 9600, 2300 RC Leiden, The Netherlands

e-mail: m.e.zonderland@lumc.nl

M. E. Zonderland · R. J. Boucherie · N. Litvak Stochastic Operations Research, University of Twente, Postbox 217, 7500 AE Enschede, The Netherlands

C. L. A. M. Vleggeert-Lankamp

Department of Neurosurgery, Leiden University Medical Center, Postbox 9600, 2300 RC Leiden, The Netherlands
Keywords Surgical scheduling • Operating rooms • Emergency patient flow • Queuing theory • Markov decision processes

\section{Introduction}

We consider a surgical department where elective, urgent and semi-urgent (synonym: semi-elective) patients are treated. An example of a department with such characteristics is a neurosurgery department. Urgent treatment is, among others, required for ruptured aneurysms, epidural or subdural hematomas, cauda equina syndrome, and (instable) spine fractures compromising the myelum or cauda equina. Semi-urgent pathologies include, among others, intracranial oncology, spine fractures with no or minimal neurological symptoms, drain dysfunctionalities, and disc herniations with unbearable pain or severe neurological deficits. Apart from these pathologies, the majority of neurosurgery patients do not require surgery within one or 2 weeks, and these are regarded as elective.

There is a definite trade-off between two major intertwined issues with respect to available surgical capacity: allocation of capacity to surgical departments and optimization of the surgical schedule within departments. On the one hand, when the target is minimal use of surgical resources, a more efficient surgical schedule may reduce the slack in the schedule, and therefore reduce the required capacity while keeping the societal costs due to patient cancellation and waiting constant. On the other hand, when the target is minimal societal costs due to patient cancellation and waiting, a more efficient surgical schedule may reduce these while keeping the allocated surgical resources constant. The 
trade-off is thus between societal costs and required surgical capacity. Allocating capacity to a surgical department usually is subject to additional constraints such as the restriction in the total available time, the time allocated to other departments, labor regulations (e.g., opening hours of the operating theater), staff restrictions (e.g., available number of surgeons), and the possibility to handle exceptions (e.g., in over-time).

In this paper we take the capacity allocated to a surgical department as a starting point. We aim for robust patient scheduling schemes. We focus on the setting of a neurosurgery department treating urgent, semi-urgent and elective patients. Urgent patients are usually treated in a separate operating room (OR), but semi-urgent patients need to be fitted in the regular OR schedule. When a semi-urgent patient arrives, an elective patient is canceled to accommodate this (prioritized) patient. The cancellation of a surgery negatively affects the patient [1]. Medical professionals tend to feel sorry for the canceled patient and aim to reschedule the surgery as soon as possible. Thus, a canceled elective patient receives a semi-urgent status, and rescheduling this surgery possibly causes the cancellation of another elective patient. This knockon effect results in a clear dependency between semiurgent patient arrivals and cancellation of elective patients in subsequent weeks.

Several strategies are known from literature to cope with non-elective patients. One strategy is to reserve a small amount of time for emergency patients for whom surgery is required on the day of arrival in each elective patient OR [2], instead of dedicating one or several ORs to emergent cases [3]. Another possibility is to determine the elective patient schedule given the expected number of emergencies [4]. In all papers reviewed in [5], acute cases have to be performed at least on the day of arrival, as opposed to the semiurgent surgeries that are studied in this paper. In both [3] and [6] the authors distinguish between emergency surgeries (which have to be performed now) and urgent surgeries (which have to be performed within a day). In [4] and [7] stochastic programming is applied to support the scheduling of add-on cases, but in both papers these cases have to be completed on the day of arrival.

In [8] the authors start from a different viewpoint and determine, using a simulation model, how many elective cases can be performed in a dedicated orthopedic trauma OR. They state that when elective patients are willing to accept that their surgery might be canceled because of an incoming trauma patient, a higher throughput can be achieved. In [9] a trade-off is made between overtime and unused OR time. The paper has an operational viewpoint, by scheduling patients on an individual level. This is similar to the methodology presented in [10], where mathematical algorithms are used to schedule individual cases in available OR blocks.

The problem setting described here shows a similarity with the news vendor problem, where at the start of each decision period for that period the available capacity is matched with the required resources, and unmatched requests are discarded at the end of the period (see e.g. [9, 11-13] for news vendor problems applied to OR problems). The news vendor problem does not incorporate scheduling of discarded requests in subsequent periods, which is precisely the problem when elective surgeries are canceled and re-scheduled in subsequent periods. Modeling this knock-on effect is the natural domain of queuing theory. In this paper, we therefore invoke the powerful theory of queues to analyze the cancellation rate of elective patients given a pre-specified surgical capacity, and the influence of canceling patients on the cancellation rate in the future.

For a surgical department with given capacity handling elective, urgent and semi-urgent patients, this paper investigates reservation schemes of OR time for semi-urgent surgeries. As the arrival pattern of semi-urgent patients is unpredictable, the reserved OR may remain unused since elective patients cannot be scheduled so shortly before their surgery. We study the trade-off between cancellations of elective surgeries due to semi-urgent surgeries, and unused OR time due to excessive reservation of OR time for semi-urgent surgeries.

In the next section we first evaluate, using a queuing theory framework, the long run OR capacity needed to accommodate every incoming semi-urgent surgery. Second, we introduce another queuing model that enables a trade-off between the cancellation rate of elective surgeries and unused OR time. In Section 3 we develop a decision support tool, based on Markov decision theory, that assists the scheduling process of elective and semi-urgent surgeries. We demonstrate our results in Section 4 with actual data obtained from a department of neurosurgery, followed by the discussion and conclusion in Section 5.

\section{Model and long term behavior}

The goal of the strategic model presented in this section is to provide an estimate for the amount of OR time that should be reserved for all semi-urgent surgeries in the long run. Therefore, we do not distinguish between 
Table 1 Notation introduced in Section 2

\begin{tabular}{ll}
\hline Symbol & Description \\
\hline$K$ & Number of slots available per OR day \\
$m$ & Total number of slots assigned to department \\
$s$ & Number of slots reserved for semi-urgent surgeries \\
$W_{n}$ & $\begin{array}{c}\text { Number of semi-urgent slots waiting for surgery } \\
\text { at the start of week } n\end{array}$ \\
$W$ & Number of semi-urgent slots waiting for surgery \\
& $\quad$ at the start of a week in a stationary regime \\
$\mathbf{q}$ & Equilibrium distribution of $W$ \\
$P_{W}(z)$ & Generating function of $W$ \\
$\lambda$ & Arrival rate of semi-urgent surgeries \\
$p_{k}$ & $\quad$ P(Surgery is of length $k$ slots), $k=1,2, . . K$ \\
$R_{n}$ & Number of semi-urgent slots that arrive during week $n$ \\
$P_{R}(z)$ & Generating function of the number of arrivals per week \\
$N_{e}$ & Number of unused reserved semi-urgent slots per week \\
$N_{c}$ & Number of canceled elective slots per week \\
$C_{e}$ & Cost of one unused reserved semi-urgent slot \\
$C_{c}$ & Cost of one canceled elective slot \\
$C_{t}$ & Total Costs \\
\hline
\end{tabular}

the 1- and 2-week streams or take overtime into account. These components of the problem are discussed in the tactical model presented in Section 3. Obviously, dynamically adjusting the amount of reserved OR time according to the effectuated inflow of semi-urgent surgeries would result in little unused OR time. However, given hospital policy that dictates that elective patients should be planned weeks in advance, such an adaptive policy would impose canceling the elective patients that were planned in the claimed slots. In order to make the trade-off between cancellation of surgeries and unused OR capacity, a constant amount of OR time is reserved for semi-urgent surgeries.

A summary of the notation used is listed in Table 1.

\subsection{Assumptions and model parameters}

The time available per OR day is divided into $K$ slots of equal length. Surgeries can have a duration of $1,2, . ., K$ slots $(K<\infty)$, and are categorized according to this duration.

When a surgery has an expected duration of more than $K$ slots, it is also included into the category of surgeries with length $K$ slots. The total number of OR slots assigned to the department per week $(m)$ equals the number of OR days per week multiplied by $K$. In order to accommodate semi-urgent patients, every week a fixed number of slots $(s)$ is reserved $(0 \leq s \leq m)$. Given the impact of the surgery on the patient and the undesirability of performing semi-urgent surgeries in overtime, we assume, in line with medical practice (see Section 1), that canceled elective patients become semi-urgent patients the following week. These patients need to undergo surgery within 1 week of their canceled surgery.

\subsubsection{Progression of the number of semi-urgent slots}

We focus on the number of semi-urgent slots waiting at the start of week $n\left(W_{n}\right)$. This equals the amount of semi-urgent slots that arrived during the previous week $\left(R_{n-1}\right)$ plus the elective slots that were canceled during the previous week in order to accommodate surplus semi-urgent slots. Elective slots are canceled if the reserved capacity for semi-urgent slots is insufficient. Recall that, in accordance with medical practice, the canceled elective slots from week $n$ become semiurgent slots in week $n+1$. Therefore, for our analysis of $W_{n}$, elective slots are not canceled, but instead the surplus semi-urgent slots from week $n$ are transferred to week $n+1$. An example of the progression in the number of semi-urgent slots waiting at the start of week $n$ is given in Fig. 1.

\subsubsection{The arrival process}

The number of arriving semi-urgent slots per week is equal to the sum of the number of slots per arriving patient. Patients arrive independently according to a Poisson process, furthermore the number of slots per arriving patient is random. Therefore we can model the arrival process with the compound Poisson process [14]. The arrival rate of semi-urgent patients is $\lambda$. Let $p_{k}$ denote the probability that an arriving semi-urgent

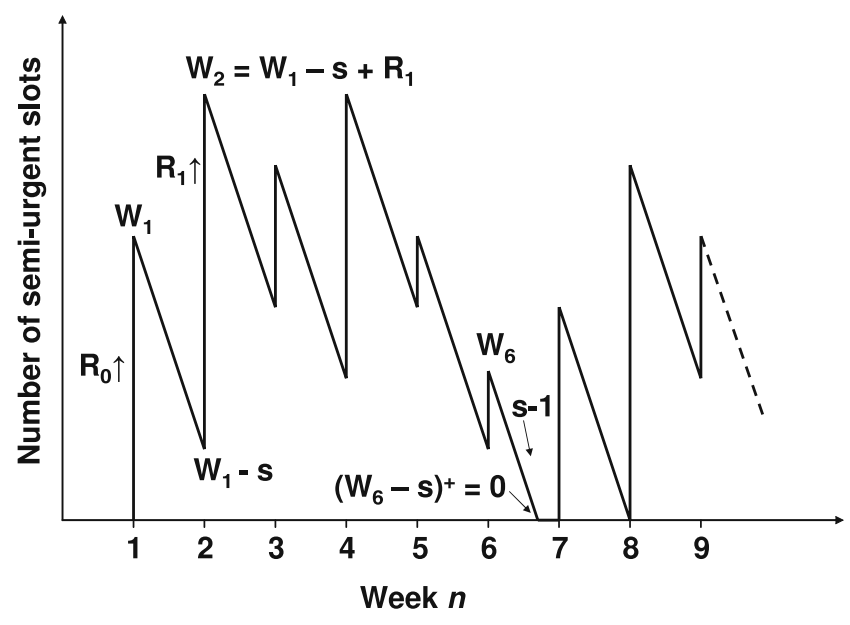

Fig. 1 An example of the progression of the number of semiurgent slots waiting at the start of the week $(s=3)$ 
surgery is of size $k$ slots, $k=1, \ldots, K$. The generating function of the arrival process is [14]:

$P_{R}(z)=\sum_{j=0}^{\infty} \mathbb{P}(R=j) z^{j}=e^{-\lambda\left(1-\sum_{k=1}^{K} p_{k} z^{k}\right)}, \quad$ where

$\sum_{k=1}^{K} p_{k}=1, \quad$ and $\quad|z| \leq 1$.

\subsection{Stability of the system}

From the description in Subsection 2.1.1 (see also Fig. 1) it is clear that the number of semi-urgent slots waiting at the start of week $n+1$ equals the number of semi-urgent slots that arrived during week $n$ plus the number of surplus semi-urgent slots of week $n$ :

$W_{n+1}=R_{n}+\left\{W_{n}-s\right\}^{+}, n=1,2, \ldots$ and $W_{1}=R_{0}$,

where $\{x\}^{+}=0$ if $x<0$ and $x$ otherwise. This is the Lindley equation for the sojourn time in a $G I / G / 1$ queue [15]. The limit for $n \rightarrow \infty$ on $W_{n+1}$ converges in distribution to $W$ if $\mathbb{E}[R]<s$, and therefore we can conclude that as long as the expected weekly amount of semi-urgent slot arrivals, $\mathbb{E}[R]$, is strictly smaller than the number of slots allocated to semi-urgent surgeries, $s$, the system is stable and the capacity reserved for these slots should be sufficient on average. It follows that there is a minimum amount of capacity $\left(s_{\min }\right)$ that should be reserved for semi-urgent surgeries: $s_{\min }=$ $\lceil\mathbb{E}[R]\rceil$, where $\lceil x\rceil$ equals $x$ rounded up to the nearest integer.

\subsection{Stationary distribution of the number of} semi-urgent slots waiting

At the start of every week the state of the system is inspected. We represent the system by a slotted queuing model in discrete time [16]. We can distinguish between two situations: (1) more semi-urgent slots are waiting than can be completed in one week (epochs 2-6 and 9 in Fig. 1), and (2) less (epoch 7 in Fig. 1) or an equal amount of semi-urgent slots are waiting (epoch 8 in Fig. 1) than can be completed. We obtain the following expressions for the transition probabilities:

$$
\begin{aligned}
\mathbb{P}\left(W_{n+1}\right. & \left.=w_{n+1} \mid W_{n}=w_{n}\right) \\
& = \begin{cases}\mathbb{P}\left(R_{n}=w_{n+1}-w_{n}+s\right) & \text { if } w_{n}-s>0 \\
\mathbb{P}\left(R_{n}=w_{n+1}\right) & \text { otherwise. }\end{cases}
\end{aligned}
$$

Define $P$ as the matrix with transition probabilities. Let $\mathbf{q}=\left(\begin{array}{lll}q_{0} & q_{1} & \cdots\end{array}\right)$ denote the equilibrium distribution of $W$, the number of semi-urgent slots waiting at the start of a week, where $q_{i}=\mathbb{P}(W=i)$. The $q_{i}$ 's can be computed as $\mathbf{q}=\mathbf{q} P$. An expression for the generating function of the equilibrium probabilities $q_{i}$ is [16]:

$P_{W}(z)=\frac{P_{R}(z) \sum_{i=0}^{s-1} q_{i}\left(z^{s}-z^{i}\right)}{z^{s}-P_{R}(z)}, \quad|z| \leq 1$,

with $P_{R}(z)$ as given in (1). To obtain an exact expression for $P_{W}(z)$ we have to determine the $s$ unknows $q_{0}, q_{1}, \ldots, q_{s-1}$. By Rouché's Theorem [17] it can be shown that the denominator of $P_{W}(z)$ has $s-1$ zeros inside the unit disk [18]. Since $P_{W}(z)$ is a generating function and therefore bounded for all $|z| \leq 1$, the zeros of the denominator are zeros of the numerator as well [16]. Thus we obtain $s-1$ equations for the $s$ unknowns $q_{0}, q_{1}, \ldots, q_{s-1}$. To derive the last equation, we use that $P_{W}(1)=1$. In order to find the $s-1$ zeros of the denominator of $P_{W}(z)$, we start by solving

$z^{s}-P_{R}(z)=0, \quad$ which is equivalent to

$$
z^{s}=e^{-\lambda \uparrow\left(1-\sum_{k=1}^{K} p_{k} z^{k}\right)}
$$

We replace this equation by $s-1$ equations, where each $z_{j}$ is a solution of the above equation [19]:

$z_{j}=F\left(z_{j}\right) e^{2 \pi \tilde{l}_{s}^{i}}, \quad$ with $\quad F(z)=e^{-\frac{\lambda}{s}\left(1-\sum_{k=1}^{K} p_{k} z^{k}\right)}$,

and $\tilde{\imath}=\sqrt{-1}$. For each value of $j(j=1,2, \ldots, s-1)$, we numerically solve this equation by using fixed point iteration [20]:

$$
\begin{aligned}
z_{j}^{(n+1)} & =F\left(z_{j}^{(n)}\right) e^{2 \pi \tilde{\imath}_{\frac{j}{s}}}, \quad n=0,1, \ldots \quad \text { and } \\
z_{j}^{(0)} & =0 .
\end{aligned}
$$

The $z_{j}$ 's that are found with this procedure are also zeros of the numerator of $P_{W}(z)$. We thus obtain $s-1$ equations for the unknowns $q_{0}, . ., q_{s-1}$ that with the added equation $P_{W}(1)=1$ define $P_{W}(z),|z| \leq 1$.

\subsection{Performance measures}

We are particularly interested in the expected number of canceled elective slots per work week $\left(\mathbb{E}\left[N_{c}\right]\right)$, and the expected number of empty reserved semi-urgent slots per work week $\left(\mathbb{E}\left[N_{e}\right]\right)$. For the latter it follows from (2) and $P_{W}(1)=P_{R}(1)=1$ that

$\mathbb{E}\left[N_{e}\right]=\sum_{i=0}^{s-1}(s-i) q_{i}=s-\mathbb{E}[R]$.

The expected number of elective slots that are canceled per week equals

$$
\begin{aligned}
\mathbb{E}\left[N_{c}\right] & =\sum_{i=s}^{\infty}(i-s) q_{i}=\sum_{i=0}^{\infty} i q_{i}-s+\sum_{i=0}^{s-1}(s-i) q_{i} \\
& =P_{W}^{\prime}(1)-\mathbb{E}[R] .
\end{aligned}
$$


Since

$$
\begin{aligned}
P_{W}^{\prime}(1)= & \mathbb{E}[R] \\
& +\frac{\sum_{i=0}^{s-1} q_{i}\left(s^{2}-i^{2}-s+i\right)-s^{2}+s+P_{R}^{\prime \prime}(1)}{2(s-\mathbb{E}[R])},
\end{aligned}
$$

where

$P_{R}^{\prime \prime}(1)=\lambda \sum_{k=1}^{K} k(k-1) p_{k}+\mathbb{E}^{2}[R]$,

we see that

$\mathbb{E}\left[N_{c}\right]=\frac{\sum_{i=0}^{s-1} q_{i}\left(s^{2}-i^{2}-s+i\right)-s^{2}+s+P_{R}^{\prime \prime}(1)}{2(s-\mathbb{E}[R])}$.

\subsection{Cost structure}

Let $C_{e}$ and $C_{c}$ be the costs of one empty semi-urgent slot and one canceled elective slot. The expected total costs then equal

$\mathbb{E}\left[C_{t}\right]=\mathbb{E}\left[N_{e}\right] C_{e}+\mathbb{E}\left[N_{c}\right] C_{c}$.

The optimal number of slots to reserve for semi-urgent surgeries $\left(s^{*}\right)$ depends on the choice of $C_{e}$ and $C_{c}$, and is the value of $s$ that minimizes $\mathbb{E}\left[C_{t}\right]$.

\section{Optimal allocation of surgery slots}

Given the stochasticity of the arrival process of semiurgent patients, there will be weeks when the allocated capacity $s^{*}$ is not sufficient. In this case the department can choose to perform the surplus semi-urgent patients this week, and cancel elective patients. On the other hand, the department can choose to postpone the semiurgent surgeries until next week. A major drawback of this operational mode is that new semi-urgent patients arrive, who together with the postponed patients from this week, pose a huge demand on available resources. Furthermore, as mentioned in the introduction, if the number of semi-urgent slots waiting for treatment exceeds the weekly amount of OR slots available, semiurgent surgeries have to be performed in overtime, which is very undesirable as well. In this section we describe a Markov decision model that provides a scheduling strategy for surplus semi-urgent slots, given the parameters obtained with the queuing model. A summary of the additional notation introduced in this section is given in Table 2 .

\subsection{Assumptions}

In this model we employ a more detailed view of the process, and consider the inflow of the two types of
Table 2 Additional notation introduced in Section 3

\begin{tabular}{ll}
\hline Symbol & Description \\
\hline$W 1_{n}$ & Number of 1-week semi-urgent slots \\
& waiting for surgery at the start of week $n$ \\
$W 2_{n}$ & Number of 2-week semi-urgent slots \\
& waiting for surgery at the start of week $n$ \\
$w_{n}=\left(w 1_{n}, w 2_{n}\right)$ & System state at start of week $n$ \\
$a_{n}$ & Action chosen in week $n$ \\
$R 1_{n}$ & Number of 1-week semi-urgent slot \\
& arrivals during week $n$ \\
$R 2_{n}$ & Number of 2-week semi-urgent slot \\
& arrivals during week $n$ \\
$\lambda_{1}$ & Arrival rate of 1-week semi-urgent \\
& surgeries \\
$\lambda_{2}$ & Arrival rate of 2-week semi-urgent \\
& surgeries \\
$p_{1 k}$ & $\mathbb{P}(1$-week semi-urgent surgery is \\
& of length $k$ slots), $k=1,2, . ., K$ \\
$p_{2 k}$ & $\mathbb{P}(2$-week semi-urgent surgery is \\
& of length $k$ slots), $k=1,2, . ., K$ \\
$N_{e, n}$ & Number of unused reserved semi-urgent \\
$N_{c, n}$ & slots during week $n$ \\
$N_{o, n}$ & Number of canceled elective slots \\
$C_{o}$ & during week $n$ \\
$C_{t, n}$ & Number of slots performed in overtime \\
$\delta_{M}$ & during week $n$ \\
& Cost of performing one slot in overtime \\
& Total costs incurred in week $n$ \\
& Discount factor \\
& Optimal policy \\
& Monotone policy \\
&
\end{tabular}

semi-urgent surgeries separately: the first type of semiurgent surgeries need to be performed within one week, the second type of semi-urgent surgeries need to be performed within two weeks. Given the system status at the beginning of week $n$, we decide how many 1and 2-week semi-urgent slots should be performed this week. Since 1-week semi-urgent surgeries have to be performed this week, all incoming surgeries of this type are scheduled for this week. First the reserved slots $\left(1,2, . ., s^{*}\right)$ are used, and if additional 1-week semiurgent demand remains, elective slots are canceled.

One-week semi-urgent demand that is still unaccommodated is performed in overtime. There are several options for scheduling 2-week patients. A logical choice would be to first schedule all 1-week slots, then schedule 2-week slots in the reserved slots of this week that are still available. Subsequently, it has to be decided whether to perform the remaining 2-week slots either this or next week. If the remaining 2-week slots are scheduled for next week, no elective slots have to be canceled this week. On the other hand, postponed 2week semi-urgent slots have evolved into 1-week semiurgent slots the next week. The existence of these slots, 
together with newly arrived 1-week semi-urgent slots, can result in a vast amount of semi-urgent demand that possibly has to be treated in overtime. In this section, a Markov decision model is presented that enables a trade-off between these two factors. For an overview of Markov decision theory, see [21]. In the model, we make the following assumptions:

- All 1-week semi-urgent slots are planned this week.

- Two week semi-urgent slots not planned this week become 1-week semi-urgent slots next week.

- Elective slots canceled this week become 2-week semi-urgent slots next week.

\subsection{The Markov decision model}

We use a Markov decision model with infinite planning horizon to support the department in deciding how many 2-week slots should be planned in a certain week (action $\left.a_{n}\right)$. The system state at the start of week $n,(n=$ $0,1, \ldots, \infty)$, is given by $w_{n}=\left(w 1_{n}, w 2_{n}\right)$, where $w 1_{n}$ and $w 2_{n}$ are the number of 1- and 2-week semi-urgent slots waiting at that moment. The action chosen depends on the number of 2-week slots waiting and on the part of capacity that is already allocated to 1-week slots. Summarizing, the range for action $a_{n}$ is determined by $\left(0,1, . ., \min \left(w 2_{n},\left(m-w 1_{n}\right)^{+}\right)\right)$.

\subsubsection{Transition probabilities}

Let the random variables $R 1_{n}$ and $R 2_{n}$ denote the number of 1- and 2-week semi-urgent slot arrivals during week $n$, where $R 1_{n}+R 2_{n}=R_{n}$. Similarly to the queuing model presented in Section $2, R 1$ and $R 2$ follow a compound Poisson distribution, with arrival rates $\lambda_{1}$ and $\lambda_{2}$, and $p_{1 k}$ and $p_{2 k}$ the probability that a 1- and 2 -week surgery is of length $k$ slots.

Recall that $m$ slots are available each week for both elective and semi-urgent surgeries. Therefore, when the number of 1-week semi-urgent slots waiting exceeds $m$, or when the sum of 1- and 2-week semi-urgent slots waiting exceeds $2 m$, the surplus semi-urgent slots are performed in overtime. Figure 2 shows how the number of slots performed in overtime is calculated. In our model, we take into account the overtime by including (high) costs for each overtime surgery slot. However, the slots performed in overtime do not affect the system state, as they have left the system in the subsequent week. Thus, the state space $A$ of the system is described as follows:

$$
\begin{aligned}
A= & \{w=(w 1, w 2): w 1, w 2=0,1, \ldots ; \\
& w 1 \leq m ; w 1+w 2 \leq 2 m\} .
\end{aligned}
$$

$W 1<m, W 1+W 2>2 m$ (area B in Figure 3):

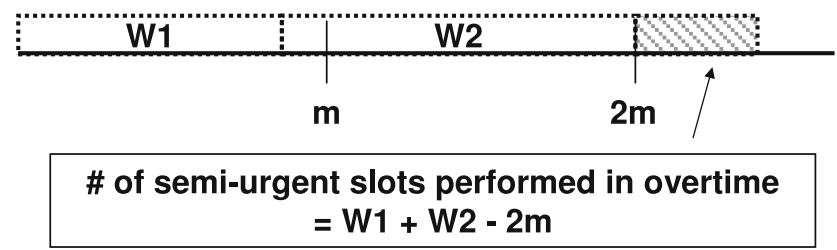

W1 > m, W2 > m (area C in Figure 3):

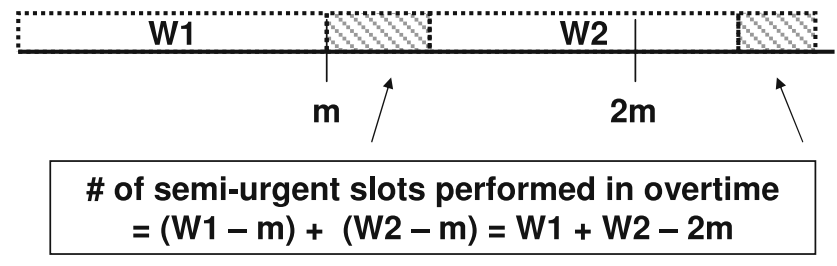

$\mathrm{W} 1>\mathrm{m}, \mathrm{W} 2<\mathrm{m}$ (area D in Figure 3):

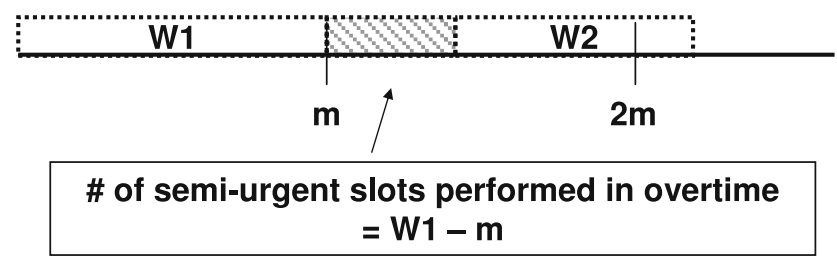

Fig. 2 Number of semi-urgent slots performed in overtime: three different cases

The state space is depicted in Fig. 3. The areas $B, C$ and $D$ and the arrows correspond to the three different cases of handling the overtime slots (see also Fig. 2). For notational purposes, let

$$
\begin{aligned}
& \mathbb{P}\left(w_{n} \mid w_{n-1}, a\right) \\
& =\mathbb{P}\left(W 1_{n}=w 1_{n}, W 2_{n}=w 2_{n} \mid W 1_{n-1}=w 1_{n-1},\right. \\
& \left.\quad W 2_{n-1}=w 2_{n-1}, a_{n-1}=a\right) .
\end{aligned}
$$

Now we define these transition probabilities for each $w_{n} \in A$.

If $w 1_{n}<m$ and $w 1_{n}+w 2_{n}<2 m$ then no slots are performed in overtime in week $n$ and thus we have

$$
\begin{aligned}
\mathbb{P}\left(w_{n} \mid w_{n-1}, a\right)= & \mathbb{P}\left(R 1_{n-1}=w 1_{n}-w 2_{n-1}+a\right) \\
& \times \mathbb{P}\left(R 2_{n-1}=w 2_{n}-\left(w 1_{n-1}-s+a\right)^{+}\right), \\
& w 1_{n}<m, w 1_{n}+w 2_{n}<2 m,
\end{aligned}
$$

and $\left(w 1_{n-1}-s+a\right)^{+}$is the number of canceled elective slots.

Now, assume that at the start of week $n$ we have $w 1$ 1-week semi-urgent slots and $w 2$ 2-week semi-urgent slots waiting. If $w=(w 1, w 2) \in B$ then some slots have to be performed in overtime as explained above. Thus, 


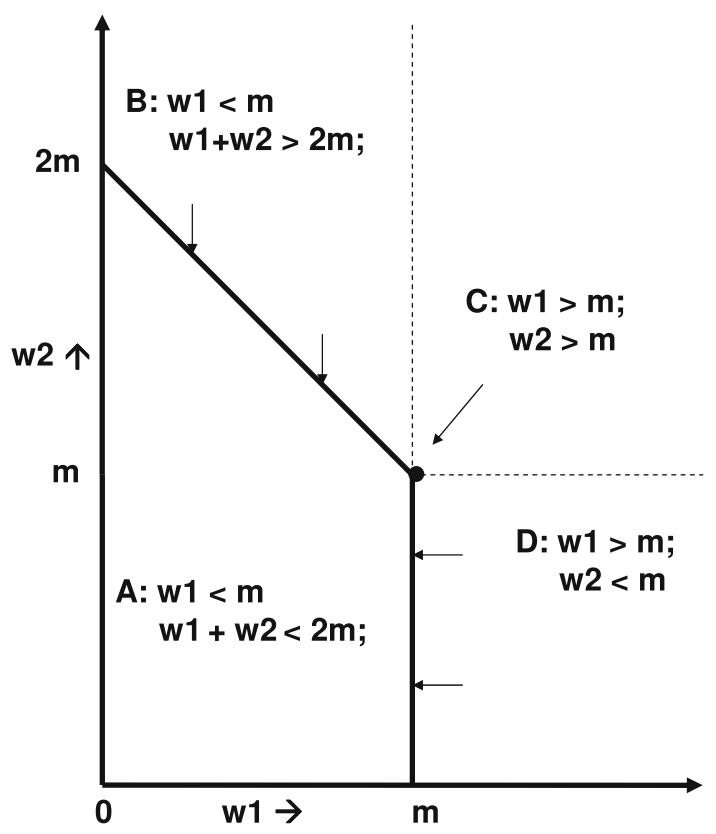

Fig. 3 State space of the system

according to the overtime policy depicted in Fig. 2, the next state is given by $w 1_{n}=w 1, w 2_{n}=2 m-w 1_{n}$, a point on the boundary between $A$ and $B$, as pointed out with arrows in Fig. 3. Including this into transition probabilities, we derive

$$
\begin{aligned}
\mathbb{P}\left(w_{n} \mid w_{n-1}, a\right)= & \mathbb{P}\left(R 1_{n-1}=w 1_{n}-w 2_{n-1}+a\right) \\
& \times \mathbb{P}\left(R 2_{n-1} \geq w 2_{n}-\left(w 1_{n-1}-s+a\right)^{+}\right), \\
w 1_{n}< & m, w 1_{n}+w 2_{n}=2 m .
\end{aligned}
$$

Analogously, if at the start of week $n$ the number of waiting semi-urgent slots is described by $w \in C$, then the next state is $w_{n}=(m, m)$, and thus the transition probabilities for this state are given by

$$
\begin{aligned}
\mathbb{P}\left(w_{n} \mid w_{n-1}, a\right)= & \mathbb{P}\left(R 1_{n-1} \geq w 1_{n}-w 2_{n-1}+a\right) \\
& \times \mathbb{P}\left(R 2_{n-1} \geq w 2_{n}-\left(w 1_{n-1}-s+a\right)^{+}\right), \\
w_{n}= & (m, m) .
\end{aligned}
$$

Finally, $w \in D$ will result in the state with $w 1_{n}=m$, and we obtain

$$
\begin{aligned}
\mathbb{P}\left(w_{n} \mid w_{n-1}, a\right)= & \mathbb{P}\left(R 1_{n-1} \geq w 1_{n}-w 2_{n-1}+a\right) \\
& \times \mathbb{P}\left(R 2_{n-1}=w 2_{n}-\left(w 1_{n-1}-s+a\right)^{+}\right), \\
w 1_{n}= & m, w 2_{n}<m .
\end{aligned}
$$

Note that $\mathbb{P}\left(R 1_{n} \leq x\right)=\mathbb{P}\left(R 2_{n} \leq x\right)=0$ if $x<0$.

\subsubsection{Performance measures}

The performance measures that were introduced for the queuing model are calculated on a weekly basis. Given the state $w_{n}=\left(w 1_{n}, w 2_{n}\right)$ and action $a$, the number of unused reserved semi-urgent slots and the number of canceled electives can be established as follows:

$N_{e, n}=\left(s-w 1_{n}-a\right)^{+}, \quad$ and

$N_{c, n}=\left(w 1_{n}-s+a\right)^{+}$.

Besides, we introduce a new performance measure, $\mathbb{E}\left[N_{o}\right]$ the expected number of semi-urgent slots that have to be performed in overtime next week as a consequence of the chosen action of this week. In week $n$, this amount depends on the number of slots at the start of week $n$, as described in Fig. 2. The formula for computing $E\left[N_{o, n+1} \mid w_{n}, a\right]$ is given in Eq. 3 .

$$
\begin{aligned}
\mathbb{E}\left[N_{o, n+1} \mid w_{n}, a\right]= & \sum_{\substack{w 1<m \\
w 1+w 2>2 m}}(w 1+w 2-2 m) \times \mathbb{P}\left(R 1_{n}=w 1-w 2_{n}+a\right) \quad \mathbb{P}\left(R 2_{n}=w 2-\left(w 1_{n}-s+a\right)^{+}\right) \\
& +\sum_{\substack{w 1>m \\
w 1+w 2>2 m}}(w 1+w 2-2 m) \times \mathbb{P}\left(R 1_{n}=w 1-w 2_{n}+a\right) \quad \mathbb{P}\left(R 2_{n}=w 2-\left(w 1_{n}-s+a\right)^{+}\right) \\
& +\sum_{\substack{w 1>m \\
w 2<m}}(w 1-m) \times \mathbb{P}\left(R 1_{n}=w 1-w 2_{n}+a\right) \quad \mathbb{P}\left(R 2_{n-1}=w 2-\left(w 1_{n}-s+a\right)^{+}\right) .
\end{aligned}
$$

\subsubsection{Cost structure}

The costs incurred for unused semi-urgent slots $\left(C_{e}\right)$ and canceled elective slots $\left(C_{c}\right)$ are equivalent with those introduced in Section 2. An extra cost, $C_{o}$, for performing 1- and 2-week slots in overtime is introduced. The expected total costs incurred in week $n$ equal

$\mathbb{E}\left[C_{t, n}\right]=\mathbb{E}\left[N_{e, n}\right] C_{e}+\mathbb{E}\left[N_{c, n}\right] C_{c}+\mathbb{E}\left[N_{o, n+1}\right] C_{o}$. 


\subsection{Determination of optimal policy}

In the process of coming to an optimal policy $\delta^{*}$ that defines an optimal action for each state $w_{n}$, we want to take into account the costs incurred today and in the future. However, we consider the costs experienced today as being more important than those experienced in the future. Therefore we use discount factor $\alpha, \alpha \in(0,1)$, in order to recalculate future costs to the cost level of today. Define $V_{\delta}\left(w_{0}\right)$ as the expected discounted costs over an infinite horizon, given initial state $w_{0}$ :

$V_{\delta}\left(w_{0}\right)=\mathbb{E}_{\delta}\left[\sum_{n=0}^{\infty} \alpha^{n} C_{t, n}\left(W_{n}, a_{n}\right) \mid w_{0}\right]$.

Let $V\left(w_{0}\right)$ denote the minimal value of $V_{\delta}\left(w_{0}\right)$ :

$V\left(w_{0}\right)=\min _{\delta} \quad V_{\delta}\left(w_{0}\right)$.

For each initial state $w_{0}$ and every action $a$, in an optimal policy it should hold that

$V\left(w_{0}\right) \leq C_{t, 0}\left(w_{0}, a_{0}\right)+\alpha \sum_{w_{1}} \mathbb{P}\left(w_{1} \mid w_{0}, a\right) V\left(w_{1}\right)$.

This gives us the optimality equation

$V\left(w_{0}\right)=\min _{a \in \delta}\left\{C_{t, 0}\left(w_{0}, a_{0}\right)+\alpha \sum_{w_{1}} \mathbb{P}\left(w_{1} \mid w_{0}, a\right) V\left(w_{1}\right)\right\}$.

The optimal policy $\delta^{*}$ consists of the values of $a$ that solve the optimality equation for each state. In order to find an optimal policy $\delta^{*}$, we use the policy iteration algorithm [22]. Since the state and action space are finite, the policy iteration algorithm converges in a finite number of steps.

Note that it is never optimal to perform 2-week slots in overtime, since even if they are postponed and then cannot be treated in regular time, they can be treated in overtime next week as well.

\section{Capacity planning and scheduling at a Department of Neurosurgery}

In this section we illustrate our modeling and optimization approach by considering a department of neurosurgery situated in an academic hospital in the Netherlands. Department staff feared that dedicating scarce OR time to the uncertain stream of semi-urgent patients would lead to an excessive amount of unused OR capacity, and therefore decided to plan almost only
Table 3 Parameter values for queuing model (Section 2)

\begin{tabular}{ll}
\hline Parameter & Value \\
\hline$\lambda$ & $11 / 2$ \\
$p_{1}$ & $29 / 55$ \\
$p_{2}$ & $11 / 55$ \\
$p_{3}$ & $15 / 55$ \\
\hline
\end{tabular}

elective patients in the available OR time. As a consequence, in daily operation, a large portion of elective surgeries was canceled in order to accommodate semiurgent surgeries. Furthermore, many ad hoc decisions were needed to ensure that all patients would receive the care they needed. Supported by our models, we show possibilities for improvement.

All surgeries performed by the department can be characterized by the estimated OR time as follows: a) one third of an OR day, b) two thirds of an OR day, c) one OR day, and d) more than one OR day. With this in mind the OR day is divided into three slots of equal length $(K=3)$. Type 1 surgeries have an estimated duration of one slot, type 2 of two slots, and type 3 surgeries an estimated duration of three or more slots. Therefore, it is either possible to perform in one OR day i) three type 1 surgeries, ii) one type 1 and one type 2 surgery, or iii) one type 3 surgery. The department is assigned eight OR days each week. With each day consisting of three slots, the department has 24 slots per week at its disposal (i.e. $m=24$ ).

\subsection{Data}

The data needed for the model, semi-urgent patient arrivals, their expected surgery duration and semiurgent state (i.e. surgery within one or two weeks) were recorded for a consecutive period of ten weeks. The characteristics of the arrival process are in line with the compound Poisson arrival process as outlined in [14]. Furthermore, the variance to mean ratio (vmr), defined as $\frac{\sigma^{2}}{\mu}$, which equals 1 for the Poisson

Table 4 Parameter values for Markov decision model (Section 3)

\begin{tabular}{lc}
\hline Parameter & Value \\
\hline$\lambda_{1}$ & $31 / 10$ \\
$\lambda_{2}$ & $12 / 5$ \\
$p_{11}$ & $20 / 31$ \\
$p_{12}$ & $5 / 31$ \\
$p_{13}$ & $6 / 31$ \\
$p_{21}$ & $9 / 24$ \\
$p_{22}$ & $6 / 24$ \\
$p_{23}$ & $9 / 24$ \\
\hline
\end{tabular}


Table 5 Cost combinations

\begin{tabular}{lrrr}
\hline Name & $C_{e}$ & $C_{c}$ & $C_{o}$ \\
\hline$C C_{1}$ & 1 & 1 & 100 \\
$C C_{2}$ & 10 & 1 & 100 \\
$C C_{3}$ & 1 & 10 & 100 \\
\hline
\end{tabular}

distribution, shows that modeling the patient arrival process at this department with a Poisson process gives a conservative estimate for the aggregated semi-urgent patient stream ( $\mathrm{vmr}=0.25$, so the variance is lower than would be expected from the Poisson distribution), while it provides a good estimate for the 1-week semiurgent patient flows $(\mathrm{vmr}=1.03)$ and a slight conservative estimate for the 2-week semi-urgent patient flow $(v m r=0.76)$. Therefore we feel confident that the compound Poisson process is an appropriate choice for modeling the arrival process of semi-urgent surgeries at this department. Table 3 gives the parameter values derived from the data, used in the queuing model. Since in the Markov decision model a distinction is made between 1- and 2-week semi-urgent surgeries, different parameter values for the compound Poisson process apply (Table 4).

The cost parameters as defined in Sections 2 and 3 should be determined by the department, and depend on the emphasis the department wants to put on either canceling patients or having an empty OR. For example, when $C_{e}=10$ and $C_{c}=1$, having an empty semi-urgent slot is considered ten times worse than canceling one elective slot. Since the department considers performing semi-urgent slots in overtime as very undesirable, we emphasize on this by fixing $C_{o}$ on 100 . We consider three combinations for $C_{e}$ and $C_{c}$ (Table 5). For the department under consideration, $C C_{1}$ is a reasonable cost configuration. To demon- strate our methodology we also use two other cost configurations.

\subsection{Determining the required number of semi-urgent} slots

We start by calculating the minimal amount of semiurgent slots required $\left(s_{\text {min }}\right)$, which is equal to $\lceil\mathbb{E}[R]\rceil$ (see Section 2.2). Since

$\mathbb{E}[R]=\lambda \sum_{k=1}^{K} k p_{k}$,

we have that $s_{\min }=\lceil 9.6\rceil=10$. The department estimated that approximately $40 \%$ of surgeries performed during regular OR days is of the semi-urgent type, which is supported by the data $\left(\frac{9.6}{24}=40 \%\right)$. Given that $s$ may vary from $s_{\min }$ to $m$, we obtain the results from Table 6 . The optimal value of $\mathbb{E}\left[C_{t}\right]$ for each cost combination is given in bold. Note the vast amount of canceled elective slots for $s=10$. This shows that focusing on the average behavior of a system can result in unsatisfactory (and maybe unexpected) system outcomes. In Fig. $4 \mathbb{E}\left[N_{e}\right]$ and $\mathbb{E}\left[N_{c}\right]$ are compared graphically. We see in Table 6 that for $C C_{1}$ the optimal value of $s^{*}$ equals 13 ( $4 \frac{1}{3}$ days), for $C C_{2}, s^{*}$ equals $11\left(3 \frac{2}{3}\right.$ days), and for $C C_{3}, s^{*}$ equals 17 ( $5 \frac{2}{3}$ days).

\subsection{Allocation of 2-week semi-urgent slots}

We now use the Markov decision model to schedule the 1- and 2-week semi-urgent slots. Our goal is to find an optimal policy that prescribes the number of 2-week semi-urgent slots to plan, given any possible system state.
Table 6 Queuing model outcomes

\begin{tabular}{lccccc}
\hline$s$ & $\mathbb{E}\left[N_{e}\right]$ & $\mathbb{E}\left[N_{c}\right]$ & $\mathbb{E}\left[C_{t}\left(C C_{1}\right)\right]$ & $\mathbb{E}\left[C_{t}\left(C C_{2}\right)\right]$ & $\mathbb{E}\left[C_{t}\left(C C_{3}\right)\right]$ \\
\hline 10 & 0.40 & 23.81 & 24.21 & 27.81 & 238.54 \\
11 & 1.40 & 5.42 & 6.82 & $\mathbf{1 9 . 4 2}$ & 55.64 \\
12 & 2.40 & 2.50 & 4.90 & 26.50 & 27.36 \\
13 & 3.40 & 1.37 & $\mathbf{4 . 7 7}$ & 35.37 & 17.14 \\
14 & 4.40 & 0.82 & 5.22 & 44.82 & 12.58 \\
15 & 5.40 & 0.51 & 5.91 & 54.51 & 10.47 \\
16 & 6.40 & 0.32 & 6.72 & 64.32 & 9.61 \\
17 & 7.40 & 0.21 & 7.61 & 74.21 & $\mathbf{9 . 4 5}$ \\
18 & 8.40 & 0.13 & 8.53 & 84.13 & 9.72 \\
19 & 9.40 & 0.08 & 9.48 & 94.08 & 10.25 \\
20 & 10.40 & 0.05 & 10.45 & 104.05 & 10.94 \\
21 & 11.40 & 0.03 & 11.43 & 114.03 & 11.74 \\
22 & 12.40 & 0.02 & 12.42 & 124.02 & 12.62 \\
23 & 13.40 & 0.01 & 13.41 & 134.01 & 13.54 \\
24 & 14.40 & 0.01 & 14.41 & 144.01 & 14.48
\end{tabular}




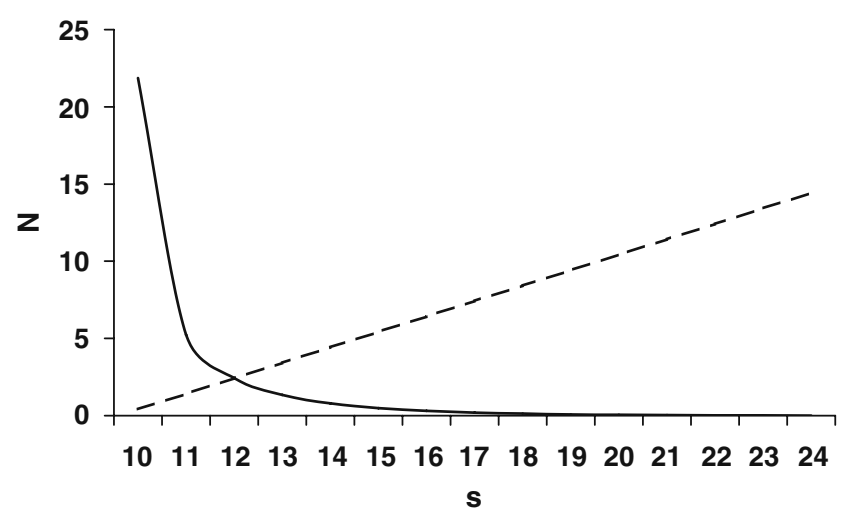

Fig. $4 \mathbb{E}\left[N_{e}\right]$ (interrupted line) and $\mathbb{E}\left[N_{c}\right]$ for $s=\left(\left\lceil s_{\min }\right\rceil, \ldots, m\right)$

\subsubsection{Monotone policy}

It is possible that in the optimal policy action $a$ is not monotone increasing in $w 2_{n}$. Although this form of the optimal policy is not uncommon in literature [23], it may be hard for medical professionals to implement. Therefore we proceed as follows. We determine an optimal policy $\delta^{*}$, as described in Section 3.3. We then check whether $a$ is monotone increasing in $w 2_{n}$. If this is the case, we maintain this optimal policy. Otherwise, we create a monotone policy, $\delta_{M}$, based on the optimal policy, where the number of 2 -week slots to plan (the chosen action) is not allowed to decrease. Such a monotone policy is not necessarily optimal, even in the class of monotone policies.

\subsubsection{Obtained policies}

The cost combinations $C C_{1}, C C_{2}$, and $C C_{3}$ are used to obtain three policies from the Markov decision model.

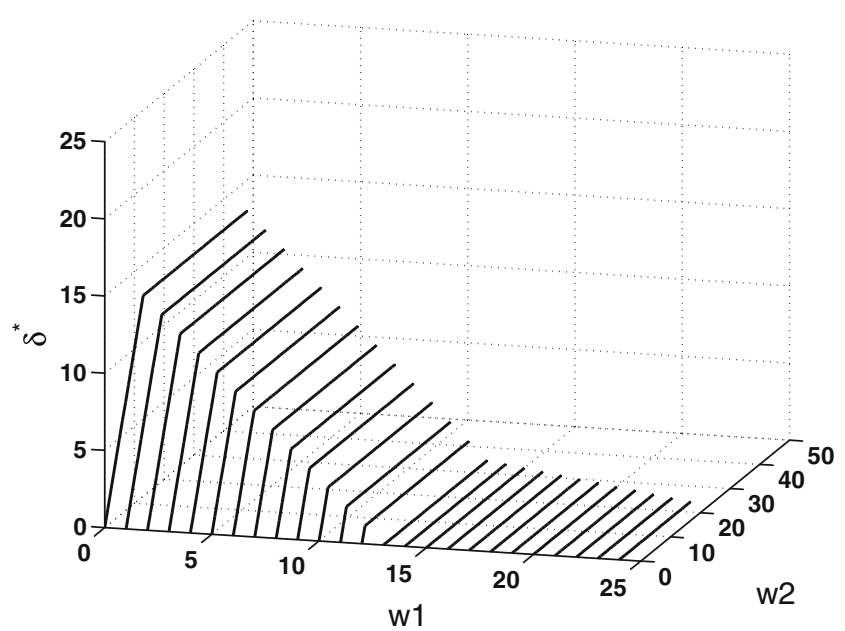

Fig. $5 \delta^{*}$ for $C C_{1}\left(s^{*}=13\right)$

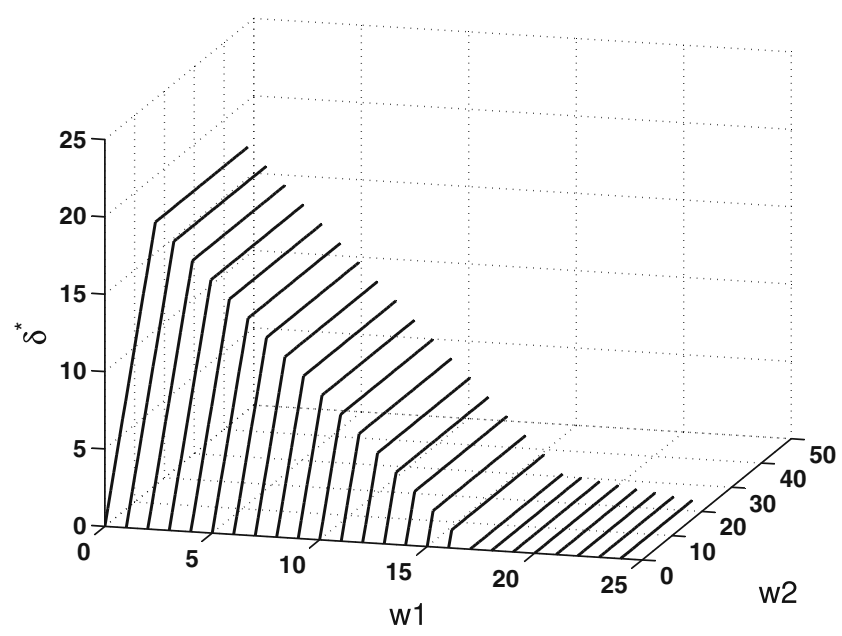

Fig. $6 \delta^{*}$ for $C C_{3}\left(s^{*}=17\right)$

For cost combinations $C C_{1}$ and $C C_{3}$ we find monotone increasing optimal policies, given in Figs. 5 and 6. For cost combination $C C_{2}$ a monotone policy was created, given in Fig. 7. A discount factor of $\alpha=0.95$ is used in all cases. We find that $\mathbb{E}\left[C_{t}\right]=4.0093$ for $C C_{1}, \mathbb{E}\left[C_{t}\right]=$ 20.2070 for $C C_{2}$, and $\mathbb{E}\left[C_{t}\right]=7.4810$ for $C C_{3}$. The horizontal axes in the figures show the possible values of $w_{1}$ and $w_{2}$. When these are combined the system state is obtained. On the vertical axis the action that is chosen for each state is given. The set of actions for all possible states forms the policy $\delta$. Recall that the action chosen consists only of the number of 2-week semi-urgent slots to plan this week, since 1-week semi-urgent slots are completed this week.

While $\delta^{*}$ for $C C_{1}$ and $C C_{3}$ is straightforward-plan 2-week slots up to $s^{*}$ and postpone the remaining 2week slots until next week, the policy obtained for $C_{2}$

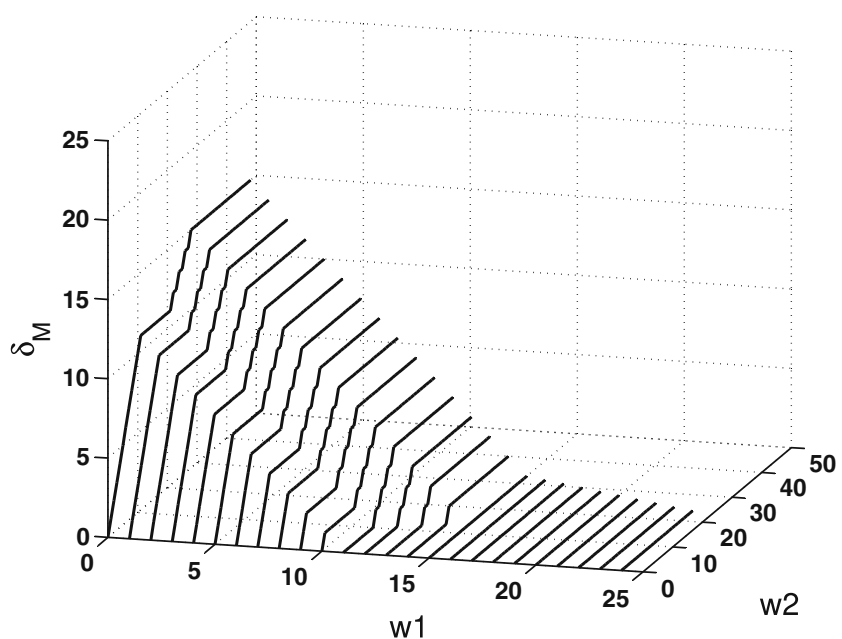

Fig. $7 \delta_{M}$ for $C C_{2}\left(s^{*}=11\right)$ 
is quite different. In several states it occurs that even when the number of 1 -week slots exceeds $s^{*}$, elective slots are canceled in order to accommodate 2-week slots. This action is chosen to avoid overtime, a result of $s^{*}$ being close to $s_{\min }$. Similar to the queuing model outcomes, this shows that maintaining a cost structure similar to $C C_{2}$, which results in choosing an $s^{*}$ which is close to $E[R]$, leads to the cancellation of elective slots.

\section{Discussion and conclusion}

In this paper we have developed a methodology to handle the semi-urgent patient flow at a surgical department. On a strategic level, we have determined the OR capacity needed to accommodate all semi-urgent patients on the long run, and we have described a queuing model that allows for a trade-off between the number of elective patients canceled and the amount of unused OR time. Given the amount of slots dedicated to semi-urgent patients, the distribution of the number of elective slots canceled, and the distribution of the number of unused semi-urgent slots can be derived with the queuing model, as is shown in Section 4. An insight that follows from these results is that focusing on only the average behavior of a system can result in undesired system outcomes, in this case the cancellation of many elective patients. Since semi-urgent patient arrivals and elective cancellations are dependent, even over consecutive periods, a natural modeling approach lies in the area of queuing theory.

On a tactical level, we have outlined a Markov decision model that supports the allocation of 1- and 2-week semi-urgent surgeries. This model provides a guideline for the weekly scheduling of semi-urgent patients. The policies obtained with the model can be transferred to a spreadsheet program and with little effort developed into a tool that is easy to use. The added value of the Markov decision model is that it simplifies the scheduling task substantially. Note that all models can be used for arbitrary parameter values.

In the methodology presented, both models involve the planning and scheduling of individual slots. It is not taken into account that when a surgery takes more than one slot, all slots must be scheduled adjacently in the same OR on the same day. To quantify this effect, we calculated the expected number of semi-urgent slots treated for the example in the case study where $s^{*}=$ 13. When considering all possible states, consisting of the number of one-, two- and three-slot semi-urgent surgeries waiting, this expectation equals 8.86 when taking into account the adjacency requirement (i.e. in the situation where we have four full OR days of three slots and a single slot on another OR day). Note that in these calculations we assumed that a rational planner would aim to maximize the number of semi-urgent slots treated in the available time. Given that we consider an instance of the problem where $s^{*}$ is relatively small, so there is little freedom to fill the OR days, the deviation of $7.7 \%$ from the value of 9.60 slots (calculated with the queuing model) will be smaller in most other (larger) instances of the problem. However, the adjency requirement results in a slightly higher demand for semiurgent slots.

A topic for further research would be to extend the presented methodology with an operational model that schedules individual surgeries. We consider the total OR time allocated to a surgical department by OR management as given. Of course, it is possible to establish the optimal amount of allocated OR time, and doing so first could result in a better performance. One of our other aims is to carry out an extensive data analysis to support an implementation of our methodology at the neurosurgery department discussed in Section 4.

Open Access This article is distributed under the terms of the Creative Commons Attribution Noncommercial License which permits any noncommercial use, distribution, and reproduction in any medium, provided the original author(s) and source are credited.

\section{References}

1. Schofield WN, Rubin GL, Piza M, Yin Lai Y, Sindhusake D, Fearnside MR, Klineberg PL (2005) Cancellation of operations on the day of intended surgery at a major Australian referral hospital. MJA 182(12):612-615

2. Wullink G, van Houdenhoven M, Hans EW, van Oostrum JM, van der Lans M, Kazemier G (2007) Closing emergency rooms improves efficiency. J Med Syst 31(6):543-546

3. Bhattacharyya T, Vrahas MS, Morrison SM, Kim E, Wiklund RA, Smith RM, Rubash HE (2006) The value of the dedicated orthopaedic trauma operating room. J Trauma Injury Infect Crit Care 60(6):1336-1341

4. Gerchak, Y, Gupta D, Henig M (1996) Reservation planning for elective surgery under uncertain demand for emergency surgery. Manag Sci 42(3):321-334

5. Cardoen B, Demeulenmeester E, Beliën J (2008) Operating room planning and scheduling: a literature review. Internal report, K.U. Leuven, Belgium. Available at https://irias. kuleuven.be/handle/123456789/165923

6. Pham DN, Klinkert A (2008) Surgical case scheduling as a generalized job shop scheduling problem. Eur J Oper Res 185(3):1011-1025

7. Lamiri M, Xie X, Dolgui A, Grimaud F (2008) A stochastic model for operating room planning with elective and emergency demand for surgery. Eur J Oper Res 185(3):1026-1037

8. Bowers J, Mould G (2004) Managing uncertainty in orthopaedic trauma theatres. Eur J Oper Res 154(3):599-608 
9. Dexter F, Macario A, O’Neill L (2000) Scheduling surgical cases into overflow block time-computer simulation of the effects of scheduling strategies on operating room labor costs. Anesth Analg 90(4):980-988

10. van Houdenhoven M, van Oostrum JM, Hans EW, Wullink G, Kazemier G (2007) Improving operating room efficiency by applying bin-packing and portfolio techniques to surgical case scheduling. Anesth Analg 105(3):707-714

11. Strum DP, Vargas LG, May JH, Bashein G (1997) Surgical suite utilization and capacity planning: a minimal cost analysis model. J Med Syst 21(5):309-322

12. McIntosh C, Dexter F, Epstein RH (2006) The impact of service-specific staffing, case scheduling, turnovers, and firstcase starts on anesthesia group and operating room productivity: a tutorial using data from an Australian hospital. Anesth Analg 103(6):1499-1516

13. Pandit JJ, Dexter F (2009) Lack of sensitivity of staffing for 8-hour sessions to standard deviation in daily actual hours of operating room time used for surgeons with long queues. Anesth Analg 108(6):1910-1915

14. Tijms HC (2003) A first course in stochastic models. Wiley, London
15. Wolff RW (1988) Stochastic modeling and the theory of queues. Prentice Hall, Englewood Cliffs, p 474

16. Bruneel H, Wuyts I (1994) Analysis of discrete-time multiserver queueing models with constant service times. Oper Res Lett 15(5):231-236

17. Kleinrock L (1975) Queueing systems, vol I: Theory. Wiley, London

18. Adan IJBF, van Leeuwaarden JSH, Winands EMM (2006) On the application of Rouché's theorem in queueing theory. Oper Res Lett 34(3):355-360

19. Bruneel H, Kim BG (1993) Discrete-time models for communication systems including ATM. Kluwer Academic, Norwell, pp 142-143

20. Atkinson KE (1978) An introduction to numerical analysis, 2nd edn. Wiley, New York

21. Puterman ML (1994) Markov decision processes: Discrete stochastic dynamic programming. Wiley, New York

22. Ross SM (1982) Introduction to stochastic dynamic programming. Academic, New York

23. Koole G (1997) Assigning a single server to inhomogeneous queues with switching costs. Theor Comp Sci 182(1-2): 203-216 